\title{
Value at Ruin and Tail Value at Ruin of the Compound Poisson Process with Diffusion and Efficient Computational Methods
}

\author{
Riccardo Gatto • Benjamin Baumgartner
}

Received: 22 March 2012 / Revised: 26 November 2012 /

Accepted: 29 November 2012 / Published online: 4 January 2013

(C) Springer Science+Business Media New York 2013

\begin{abstract}
We analyze the insurer risk under the compound Poisson risk process perturbed by a Wiener process with infinite time horizon. In the first part of this article, we consider the capital required to have fixed probability of ruin as a measure of risk and then a coherent extension of it, analogous to the tail value at risk. We show how both measures of risk can be efficiently computed by the saddlepoint approximation. We also show how to compute the stabilities of these measures of risk with respect to variations of probability of ruin. In the second part of this article, we are interested in the computation of the probability of ruin due to claim and the probability of ruin due to oscillation. We suggest a computational method based on upper and lower bounds of the probability of ruin and we compare it to the saddlepoint and to the Fast Fourier transform methods. This alternative method can be used to evaluate the proposed measures of risk and their stabilities with heavy-tailed individual losses, where the saddlepoint approximation cannot be used. The numerical accuracy of all proposed methods is very high and therefore these measures of risk can be reliably used in actuarial risk analysis.
\end{abstract}

Keywords Coherent measure of risk - Daniels' exponent • Fast Fourier transform • Lundberg's exponent - Probability of ruin - Probabilites of ruin due to claim and to oscillation • Richardson's extrapolation • Saddlepoint approximation • Stability • Upper and lower bounds

AMS 2000 Subject Classifications 41 A60 • 65C50 • 60G51

R. Gatto $(\bowtie) \cdot$ B. Baumgartner Institute of Mathematical Statistics and Actuarial Science, University of Bern, Alpeneggstrasse 22, 3012 Bern, Switzerland e-mail: gatto@stat.unibe.ch 


\section{Introduction}

The risk process is a stochastic model for the evolution of the insurance company's reserve. We consider the compound Poisson risk process perturbed by a Wiener process defined as follows. Let $x \geq 0$ denote the initial reserve, $c>0$ the constant premium rate and $\left\{Z_{t}\right\}_{t \geq 0}$ the $\mathbb{R}_{+}$-valued compound Poisson process of the aggregate claim amounts, then the compound Poisson risk process with added diffusion is given by

$$
Y_{t}=x+c t-Z_{t}+\sigma W_{t},
$$

for all $t \geq 0$, where $\left\{W_{t}\right\}_{t \geq 0}$ is a $\mathbb{R}$-valued standard Wiener process and $\sigma>0$ the associated volatility. In particular, $Z_{t}=\sum_{i=0}^{N_{t}} X_{i}$, for all $t>0$, where $X_{0} \stackrel{\text { def }}{=} 0$, for convenience, and $X_{1}, X_{2}, \ldots>0$ are independent individual claim amounts with common cumulative distribution function (CDF) $F$. The individual claim amounts are assumed independent of the Poisson process $\left\{N_{t}\right\}_{t \geq 0}$ with intensity $\lambda>0$. Further, we assume that $\mu=\mathbb{E}\left[X_{1}\right] \in(0, \infty)$. Two practical quantities related to the risk process are the relative security loading $\beta=\left(c-\mathbb{E}\left[Z_{1}\right]\right) / \mathbb{E}\left[Z_{1}\right]=c /(\lambda \mu)-1$ and $\zeta=2 c / \sigma^{2}$. The event that the surplus process ever falls below zero is called ruin and its probability is given by

$$
\psi(x)=\mathbb{P}\left(\inf _{t \geq 0} Y_{t}<0\right) .
$$

We also define the time of ruin by

$$
T= \begin{cases}\inf \left\{t>0: Y_{t} \leq 0\right\}, & \text { if the infimum exists, } \\ 0, & \text { otherwise. }\end{cases}
$$

The probability of ruin can be decomposed as $\psi(x)=\psi^{(1)}(x)+\psi^{(2)}(x)$, where $\psi^{(1)}(x)=\mathbb{P}\left(T<\infty, Y_{T}=0\right)$ is the probability that the zero line is first crossed by an oscillation of the path of the risk process, i.e. by creeping, and $\psi^{(2)}(x)=$ $\mathbb{P}\left(T<\infty, Y_{T}<0\right)$ is the probability that the zero line is first crossed by a jump of the path risk process, i. e. by an individual claim amount. By the regularity of the Wiener process, we have $\psi(0)=\psi^{(1)}(0)=1$ and thus $\psi^{(2)}(0)=0$. In general, there are no closed-form formulae for $\psi(x), \psi^{(1)}(x)$, or $\psi^{(2)}(x)$. There are however exact formulae for $\psi(x)$ when $F$ is a phase-type CDF, see Dufresne and Gerber $(1989,1991)$; Asmussen and Rolski (1991) and Neuts (1981).

In this article, we first suggest the capital required to obtain a fixed probability of ruin, typically very small, as a measure of the insurer's risk. We call this capital the value at ruin $(\mathrm{VaRu})$. In order to fulfill the coherency property of measures of risk, we then suggest an extension analogous to tail value at risk that we call tail value at ruin (TVaRu). Both measures of risk appear in Cheridito et al. (2006, Section 5.1) and in Trufin et al. (2009) in context of the standard risk process, without perturbation, but our aim is show their practical relevance when associated with effective computational techniques. We show how both new measures of risk, VaRu and TVaRu, can be efficiently computed by the saddlepoint approximation. Two auxiliary quantities to $\mathrm{VaRu}$ and $\mathrm{TVaRu}$ are their relative stabilities with respect to (w. r. t.) variations of the selected probability of ruin. We show how to compute them with the saddlepoint approximation. Although we do not quantify theoretically the degree of accuracy of all the proposed saddlepoint approximations, we illustrate by 
a numerical example that all saddlepoint approximations presented are numerically accurate. In the second part of this article, we are interested in the computation of the probability of ruin due to oscillation $\psi^{(1)}(x)$ and of the probability of ruin due to claim $\psi^{(2)}(x)$. For this purpose, we propose using Richardson's method of numerical differentiation together with a recursive method for computing upper and lower bounds of the probability of ruin. We compare numerically this new method with the saddlepoint approximation and with the Fast Fourier transform (FFT). The method of upper and lower bound together with the proposed Richardson's approximation to $\psi^{(1)}(x)$ can also be used to evaluate the $\mathrm{VaRu}$, the TVaRu and their stabilities with heavy-tailed individual claim amount CDF $F$, where the previous methods using the saddlepoint approximation do not hold anymore.

The re-expression of the probability of ruin in terms of the distribution of the maximal aggregate loss is the central part of the methods presented in Sections 2 and 3. The aggregate loss Lévy process $\left\{L_{t}\right\}_{t \geq 0}$ is defined by $L_{t}=Z_{t}-c t-\sigma W_{t}$, for all $t \geq 0$, and the maximal aggregate loss by

$$
L=\sup _{t \geq 0}\left\{L_{t}\right\}
$$

It follows that

$$
\psi(x)=\mathbb{P}(L>x)
$$

for all $x \geq 0$ and that $L$ has a proper absolutely continuous distribution if $\sigma>0$, as $\mathbb{P}(L=0)=0$ in this case. Consider the decomposition

$$
L=\sum_{i=0}^{N}\left(L_{i}^{(1)}+L_{i}^{(2)}\right),
$$

with $L_{0}^{(2)} \stackrel{\text { def }}{=} 0$ for convenience, where $N$ denotes the number of record highs of $\left\{L_{t}\right\}_{t \geq 0}$ that are caused by occurrence of claims, $L_{i}^{(1)}>0, i \in\{0, \ldots, N\}$, are determined by the records of $\left\{L_{t}\right\}_{t \geq 0}$ arising from the oscillations and $L_{i}^{(2)}>0, i \in\{1, \ldots, N\}$, are determined by the records arising from the claims, see e.g. Fig. 1 in Gatto and Mosimann (2012). Precisely, let $T_{1}, \ldots, T_{N}$ denote the occurrence times of records due to claims, and assume for convenience $T_{0} \stackrel{\text { def }}{=} 0$ and $T_{N+1} \stackrel{\text { def }}{=} \infty$. Then

$$
L_{i}^{(1)}=\sup \left\{L_{t}: T_{i}<t<T_{i+1}\right\}-L_{T_{i}},
$$

for $i \in\{0, \ldots, N\}$, and

$$
L_{i}^{(2)}=L_{T_{i}}-\left(L_{T_{i-1}}+L_{i-1}^{(1)}\right),
$$

for $i \in\{1, \ldots, N\}$. As $\left\{L_{t}\right\}_{t \geq 0}$ is a Lévy process and $T_{1}, T_{2}, \ldots$ are stopping times, we have that: $L_{0}^{(1)}, L_{1}^{(1)}, \ldots$ are independent and identically distributed (i.i.d.), $L_{1}^{(2)}, L_{2}^{(2)}, \ldots$ are i.i. d., $N$ has the geometric distribution

$$
\mathbb{P}(N=n)=(1-p)^{n} p,
$$

for all $n \in\{0,1, \ldots\}$, where $p=\beta /(1+\beta)$ is the probability that there are no record highs caused by claims, and that all these random variables are independent. Then, 
Dufresne and Gerber (1991, Section 5) show that the decomposition in Eq. 4 leads to the following series representation for the probability of ruin,

$$
1-\psi(x)=\sum_{n=0}^{\infty}(1-p)^{n} p\left(H_{1}^{*(n+1)} * H_{2}^{* n}\right)(x),
$$

where $H_{1}(x)=1-\mathrm{e}^{-\zeta x}$ and $H_{2}(x)=\int_{0}^{x} 1-F(y) \mathrm{d} y / \mu$, for all $x \geq 0$, are the CDF of $L_{1}^{(1)}$ and $L_{1}^{(2)}$, respectively.

The rest of this article has the following structure. In Section 2.2 we suggest using the capital required to reach a fixed probability of ruin, the $\mathrm{VaRu}$, as a measure of risk and we also suggest an extension of it which satisfies the coherency axioms, the TVaRu. We then provide saddlepoint approximations to the proposed risk measures and also to their stabilities, w.r.t. variations of the fixed probability of ruin. In Section 2.2 we illustrate the high numerical accuracy all proposed saddlepoint approximations. In Section 3.1 we extend a numerical method for computing upper and lower bounds to the probability of ruin, to the computation of the probability of ruin by creeping. In Section 3.2 we compare numerically this new method with the FFT and with a saddlepoint approximation. Some conclusions follow in Section 3, including a remark regarding the computation of the measures of risk and their stabilities presented in Section 2.1 when the individual claim amounts are heavy-tailed.

\section{A Coherent Measure of Risk for the Infinite Time Horizon}

In Section 2.1 we define the $\mathrm{VaRu}$, the TVaRu and their stabilities w. r.t. variations of their ruin probability level. We then provide saddlepoint approximations to these values. In Section 2.2 we illustrate the numerical accuracy of the proposed methods.

\subsection{Value and Tail Value at Ruin and their Saddlepoint Approximations}

Let $Z \geq 0$ denote a random loss for the insurer. A measure of risk is a $\mathbb{R}_{+}$-valued operator $\rho$ defined on the space of non-negative random variables, such that $\rho(Z) \geq$ 0 represents an index of the capital required for protecting against the random loss $Z$. The well-known value at risk $(\mathrm{VaR})$ of $Z$ at level $\varepsilon \in(0,1)$ is the $\varepsilon$-quantile of the distribution of $Z$, denoted $q_{Z, \varepsilon}$, where $\varepsilon$ is typically close to 1 (e.g. 0.99). The tail value at risk (TVaR) of $Z$ at level $\varepsilon$ is the conditional expected value of $Z$ beyond the VaR, precisely it is given by $\mathbb{E}\left[Z \mid Z>q_{Z, \varepsilon}\right]$, where $\varepsilon$ is again close to 1 . TVaR is also known as conditional value at risk (CVaR), average value at risk (AVaR), expected tail loss (ETL) or expected shortfall (ES). Clearly, the TVaR makes fuller use of the information provided by the right tail of the loss distribution than the VaR. But the TVaR is also a coherent measure of risk in the following sense. A measure of risk $\rho$ is called coherent if, for any two random financial losses $Z_{1}, Z_{2} \geq 0$ and for all $c>0$, it satisfies

(i) $\rho\left(Z_{1}+Z_{2}\right) \leq \rho\left(Z_{1}\right)+\rho\left(Z_{2}\right)$

(ii) $Z_{1} \leq Z_{2}$ a.s. $\Longrightarrow \rho\left(Z_{1}\right) \leq \rho\left(Z_{2}\right)$

(subadditivity),

(iii) $\rho\left(c \bar{Z}_{1}\right)=c \rho\left(Z_{1}\right)$ (monotonicity),

(iv) $\rho\left(c+Z_{1}\right)=c+\rho\left(Z_{1}\right)$ (positive homogeneity), and (translation invariance). 
For further details, see Artzner et al. (1997) or Klugman et al. (2008, Section 3.5). While TVaR is a coherent measure of risk, $\mathrm{VaR}$ is not one, because it does not fulfill the subadditivity property (i). Because subadditivity represents an important property in the context of insurance, TVaR seems a more sensible measure of risk than VaR. There are various ways of modelling the insurer's aggregate loss. One can consider an aggregate loss process at a fixed time and compute a measure of risk at that time. Gatto (2011) provides saddlepoint approximations to VaR and TVaR for the doubly compound and perturbed insurer loss process, based on a primary counting birth process, for the number of primary events (e.g. catastrophes), and on a secondary counting distribution, for the number of individual losses generated from each event of the primary process. Gatto (2012) provides a saddlepoint approximation to the VaR of compound Poisson processes with periodic intensity functions and under constant force of interest.

The previous measures of risk usually apply to a random loss at a single time. Now we want to measure the risk incurred over the whole infinite time horizon, taking into account the dynamic of the perturbed risk process. We consider the perturbed risk process in Eq. 1 and suggest the minimal initial capital required to obtain a probability of ruin smaller than or equal to a fixed threshold (possibly close to zero) as the measure of risk. We have thus the following definition.

Definition 2.1 (Value at ruin) Consider the perturbed compound Poisson risk process in Eq. 1 and its probability of ruin $\psi(x)=\mathbb{P}(T<\infty)$, for any initial capital $x \geq 0$. The associated value at ruin $(\mathrm{VaRu})$ at level $\varepsilon \in(0,1)$ is given by

$$
\operatorname{VaRu}(\varepsilon)=\inf \{x \geq 0: \psi(x) \leq \varepsilon\} .
$$

Although the $\mathrm{VaRu}$ represents an intuitive infinite time horizon measure of risk, it is not subadditive, in the sense of (i) above, and thus not coherent. In analogy to the TVaR, we can obtain a coherent measure of risk from the VaRu by considering the expected maximal aggregate loss given that the maximal aggregate loss exceeds a fixed $\mathrm{VaRu}$. This gives the following definition.

Definition 2.2 (Tail value at ruin) Consider the perturbed compound Poisson risk process in Eq. 1. Then the associated tail value at ruin (TVaRu) at level $\varepsilon \in(0,1)$ is given by

$$
\operatorname{TVaRu}(\varepsilon)=\mathbb{E}[L \mid L>\operatorname{VaRu}(\varepsilon)],
$$

where $L$ is the infinite time horizon maximal aggregate loss defined by Eq. 3 .

Thus, $\mathrm{VaRu}$ and $\mathrm{TVaRu}$ derive from the dynamic of the reserve process, over the infinite time horizon.

An important problem is the efficient computation of the $\mathrm{VaRu}$ and the TVaRu. We suggest computing these new measures of risk by the saddlepoint approximation. The saddlepoint approximation is a classical method of asymptotic analysis for approximating various types of complex integrals. It was introduced into statistics by Daniels (1954) for approximating the density of the sample mean of i.i.d. random variables. Lugannani and Rice (1980) provide a practical saddlepoint approximation to the corresponding CDF. For general references see e.g.: Barndorff-Nielsen and Cox (1989); Field and Ronchetti (1990) and Jensen (1995). Some recent applications 
of the saddlepoint approximation in actuarial models are: Gatto (2010, 2012), for the distribution of the inhomogeneous and discounted compound Poisson aggregate claim amount; Barndorff-Nielsen and Schmidli (1995), for finite and infinite time horizon probabilities of ruin in the classical risk process; and Gatto and Mosimann (2012), for the probability of ruin, the probability of ruin due to claim and the probability of ruin due to oscillation in the perturbed risk process. Combining the results of Wang (1995) and Gatto and Mosimann (2012), we now provide NewtonRaphson-type saddlepoint approximations to the VaRu and the TVaRu just defined.

Result 2.3 (Saddlepoint approximations to the VaRu) Consider the cumulantgenerating function (CGF)

$$
K_{L}(v)= \begin{cases}\log \frac{\lambda \mu \beta v}{(1+\beta) \lambda \mu v-\frac{1}{2} \sigma^{2} v^{2}+\lambda-\lambda M_{X}(v)}, & \text { if } v \neq 0, \\ 0, & \text { if } v=0\end{cases}
$$

where $\sigma>0$ and assume $M_{X}(v)=\mathbb{E}\left[\mathrm{e}^{v X_{1}}\right]$ is finite for all $v$ in a neighborhood of the origin (i. e. $X_{1}$ is light-tailed). Assume further that Lundberg's exponent (or the adjustment coefficient) $\bar{v}$, defined as the positive solution in $v$ of

$$
\lambda M_{X}(v)+\frac{1}{2} \sigma^{2} v^{2}-c v-\lambda=0,
$$

exists. Let $x>0$ and $v_{x} \in(-\infty, \bar{v})$ be Daniels' exponent (or the saddlepoint), defined as the solution in $v$ of

$$
K_{L}^{\prime}(v)=x
$$

Further let

$$
\begin{aligned}
& r_{x}=\operatorname{sgn}\left(v_{x}\right) \sqrt{2\left(v_{x} K_{L}^{\prime}\left(v_{x}\right)-K_{L}\left(v_{x}\right)\right)}, \\
& s_{x}=v_{x} \sqrt{K_{L}^{\prime \prime}\left(v_{x}\right)}
\end{aligned}
$$

and

$$
z_{x}=r_{x}+\frac{1}{r_{x}} \log \frac{s_{x}}{r_{x}} .
$$

Because Eq. 10 gives $x$ as a function of $v, z_{x}$ above can be re-expressed as a function of $v$ as well and in this case it is denoted $z(v)$. In the following, $\varepsilon \in(0,1)$ is a fixed and typically small probability of ruin.

(a) Consider

$$
q_{0}(1-\varepsilon)=-\frac{1}{\bar{v}} \log \varepsilon
$$

and the iterations

$$
q_{k}(1-\varepsilon)=q_{k-1}(1-\varepsilon)+\frac{\left[\Phi^{(-1)}(1-\varepsilon)\right]^{2}-z_{q_{k-1}(1-\varepsilon)}^{2}}{2 v_{q_{k-1}(1-\varepsilon)}}
$$


for $k \in\{1,2, \ldots\}$, where $\phi$ and $\Phi$ denote the standard normal density and CDF, respectively. If $k^{\star}$ is the smallest integer such that $\left|q_{k^{\star}}(1-\varepsilon)-q_{k^{\star}-1}(1-\varepsilon)\right|$ is smaller than a fixed threshold, then

$$
\operatorname{VaRu}_{\mathrm{S}}^{\dagger}(\varepsilon) \stackrel{\text { def }}{=} q_{k^{\star}}(1-\varepsilon)
$$

is a Newton-Raphson saddlepoint approximation to $\operatorname{VaRu}(\varepsilon)$.

(b) Consider

$$
u_{0}(1-\varepsilon)=\bar{v}\left(1+\frac{1}{\log \varepsilon}\right)
$$

the initial value

$$
u_{1}(1-\varepsilon)=u_{0}(1-\varepsilon)+\frac{\left[\Phi^{(-1)}(1-\varepsilon)\right]^{2}-z^{2}\left(u_{0}(1-\varepsilon)\right)}{2 q_{0}(1-\varepsilon)}
$$

and the iterations

$$
u_{k}(1-\varepsilon)=u_{k-1}(1-\varepsilon)+\frac{\left[\Phi^{(-1)}(1-\varepsilon)\right]^{2}-z^{2}\left(u_{k-1}(1-\varepsilon)\right)}{2 u_{k-1}(1-\varepsilon) K_{L}^{\prime \prime}\left(u_{k-1}(1-\varepsilon)\right)},
$$

for $k \in\{2,3, \ldots\}$. If $k^{\star}$ is the smallest integer such that $\mid u_{k^{\star}}(1-\varepsilon)-u_{k^{\star}-1}(1-$ $\varepsilon) \mid$ is smaller than a fixed threshold, then

$$
\operatorname{VaRu}_{\mathrm{S}}(\varepsilon) \stackrel{\text { def }}{=} K_{L}^{\prime}\left(u_{k^{\star}}(1-\varepsilon)\right)
$$

is a faster Newton-Raphson saddlepoint approximation to $\operatorname{VaRu}(\varepsilon)$.

Numerical comparisons within the example of Section 2.2 show that the two Newton-Raphson saddlepoint approximations $\mathrm{VaRu}_{\mathrm{S}}^{\dagger}$ and $\mathrm{VaRu}_{\mathrm{S}}$, given under parts (a) and (b) of Result 2.3, yield comparable relative errors and none of them is systematically better than the other one. However the computation of $\mathrm{VaRu}_{\mathrm{S}}$ never requires solving the saddlepoint equation, see Eq. 10, and in this sense it is computationally more efficient than $\mathrm{VaRu}_{\mathrm{S}}^{\dagger}$. For these reasons, only the results of $\mathrm{VaRu}_{\mathrm{S}}$ are presented in the example of Section 2.2.

Proof The function $K_{L}$ in Eq. 8 is the CGF of the maximal aggregate loss $L$ and its derivation can be found in Gatto and Mosimann (2012, Section 2). Let

$$
v_{0}= \begin{cases}\sup \left\{v \in \mathbb{R}: M_{L}(v)<\infty\right\}, & \text { if the supremum exists, } \\ \infty, & \text { otherwise. }\end{cases}
$$

Then $v_{0} \in[0, \infty]$ and the moment-generating function (MGF) $M_{L}=\exp K_{L}$ or the CGF $K_{L}$ are called steep if $\lim _{v \uparrow v_{0}} M_{L}(v)=\infty$. As in Dufresne and Gerber (1991, Eq. 7.2), we define Lundberg's exponent $\bar{v}$ of the perturbed risk process in Eq. 1 as the positive solution in $v$ of $\mathbb{E}\left[\mathrm{e}^{v\left(x-Y_{1}\right)}\right]=1$, which is equivalent to Eq. 9. After simplifications, $\bar{v}$ becomes the positive solution in $v$ of Eq. 9. Comparing Eq. 9 with the denominator of $K_{L}$ in Eq. 8, shows that $K_{L}$ is steep with steepness point $v_{0}=\bar{v}$. From the strict convexity and the differentiability of $K_{L}$ follows that $\lim _{v \uparrow \bar{v}} K_{L}^{\prime}(v)=\infty$ as well. Viewed as a function of $x \geq 0$, Daniels' exponent $v_{x}$ is thus a real-valued function, strictly increasing with $\lim _{x \rightarrow \infty} v_{x}=\bar{v}$. Note that a trivial sufficient condition for the existence of Lundberg's exponent $\bar{v}$ is provided by the steepness of $M_{X}$. 
Lugannani and Rice's saddlepoint approximation to the probability ruin is given by

$$
\psi_{\mathrm{S}}(x)=1-\Phi\left(r_{x}\right)-\left(\frac{1}{r_{x}}-\frac{1}{s_{x}}\right) \phi\left(r_{x}\right),
$$

and, from steepness, it exists for any initial reserve $x \geq 0$. The approximation to $\psi(x)$ given by

$$
\psi_{\mathrm{S}}^{*}(x)=1-\Phi\left(z_{x}\right),
$$

where $z_{x}$ is given by Eq. 13, is known having the same accuracy as Lugannani and Rice's saddlepoint approximation in Eq. 20, see Jensen (1992, Lemma 2.1). However, solving

$$
\psi_{S}^{*}(x)=1-\varepsilon \quad \Longleftrightarrow \quad z_{x}=\Phi^{(-1)}(1-\varepsilon)
$$

w. r. t. $x$ is substantially simpler than solving $\psi_{S}(x)=1-\varepsilon$ w.r.t. $x$. Both saddlepoint approximations under (a) and (b) are Newton-Raphson iterations based on Eq. 22.

Regarding part (a), the values $q_{k}(1-\varepsilon)$, for $k \in\{1,2, \ldots\}$, are derived from an expansion of $z_{x}^{2}$ equated to $\left[\Phi^{(-1)}(1-\varepsilon)\right]^{2}$, applied successively to $x=q_{0}(1-\varepsilon)$, given by Eq. 14, and to $x=q_{k}(1-\varepsilon)$, given by Eq. 15, for $k \in\{1,2, \ldots\}$. The precise developments can be found in Wang (1995, Section 2.1). The first approximation $q_{0}(1-\varepsilon)$ in Eq. 14 follows from Cramér-Lundberg's approximation $\psi(x) \sim c_{x} \mathrm{e}^{-\bar{v} x}$, where $c_{x} \rightarrow 1$ as $x \rightarrow \infty$.

The Newton-Raphson saddlepoint approximation under (b) is analogous to the one under (a). The difference is that the Newton-Raphson steps are carried out in the frequency domain, i. e. Eq. 22 is re-expressed as $z(v)=\Phi^{(-1)}(1-\varepsilon)$ and inverted w.r.t. the saddlepoint $v$. More details can be found in Wang (1995, Section 2.2). The first approximation $u_{0}(1-\varepsilon)$ in Eq. 16 is the saddlepoint of an exponential distribution with parameter $\bar{v}$ evaluated at Cramér-Lundberg's approximation in Eq. 14, precisely it is the solution in $v$ of

$$
\frac{\mathrm{d}}{\mathrm{d} v} \log \frac{\bar{v}}{\bar{v}-v}=-\frac{1}{\bar{v}} \log \varepsilon .
$$

We now give a saddlepoint approximation to the coherent measure of risk TVaRu.

Result 2.4 (Saddlepoint approximation to the TVaRu) Assume $M_{X}(v)$ finite for all $v$ in a neighborhood of the origin and that Lundberg's exponent, defined by Eq. 9, exists. Then for any given level $\varepsilon \in(0,1)$,

$$
\operatorname{TVaRu}_{\mathrm{S}}(\varepsilon)=\frac{1}{\varepsilon} \int_{u_{k^{\star}}(1-\varepsilon)}^{\bar{v}} \psi_{\mathrm{S}}\left(K^{\prime}(v)\right) K_{L}^{\prime \prime}(v) \mathrm{d} v+\operatorname{VaRu}_{\mathrm{S}}(\varepsilon)
$$

is a saddlepoint approximation to $\operatorname{TVaRu}(\varepsilon)$, where $u_{k^{\star}}(1-\varepsilon)$ is the NewtonRaphson approximation to the saddlepoint given by Eq. 18 and $K_{L}$ is the cGF of $L$ defined in Eq. 8 . 
Proof Let $\varepsilon>0$. By partial integration we obtain the integral representation of the $\mathrm{TVaRu}$ given by

$$
\operatorname{TVaRu}(\varepsilon)=\mathbb{E}[L \mid L>\operatorname{VaRu}(\varepsilon)]=\frac{1}{\varepsilon} \int_{\operatorname{VaRu}(\varepsilon)}^{\infty} \psi(x) \mathrm{d} x+\operatorname{VaRu}(\varepsilon) .
$$

The above representation and a change of integration variable leads to the saddlepoint approximation to TVaRu given by

$$
\begin{aligned}
\operatorname{TVaRu}_{\mathrm{S}}(\varepsilon) & =\frac{1}{\varepsilon} \int_{\operatorname{VaRu}_{\mathrm{S}}(\varepsilon)}^{\infty} \psi_{\mathrm{S}}(x) \mathrm{d} x+\operatorname{VaRu}_{\mathrm{S}}(\varepsilon) \\
& =\frac{1}{\varepsilon} \int_{u_{k^{\star}(1-\varepsilon)}}^{\bar{v}} \psi_{\mathrm{S}}\left(K_{L}^{\prime}(v)\right) K_{L}^{\prime \prime}(v) \mathrm{d} v+\operatorname{VaRu}_{\mathrm{S}}(\varepsilon) .
\end{aligned}
$$

The upper integration bound of the last integral is obtained from the steepness of $K_{L}$, as explained in the proof of Result 2.3.

It is often important to assess the stabilities of $\mathrm{VaRu}$ and $\mathrm{TVaRu}$ w.r.t. the ruin level, i.e. the variations of these quantities w.r.t. small variations of the level $\varepsilon \in$ $(0,1)$. We have thus the following definitions.

Definition 2.5 (Value at ruin stability) Consider the perturbed compound Poisson risk process in Eq. 1 and its probability of ruin $\psi(x)=\mathbb{P}(T<\infty)$, for any $x \geq 0$. Assume $\psi$ is strictly decreasing at level $\varepsilon \in(0,1)$. Then the associated value at ruin stability $(D \mathrm{VaRu})$ at level $\varepsilon \in(0,1)$ is given by

$$
D \operatorname{VaRu}(\varepsilon)=\left.\frac{\mathrm{d}}{\mathrm{d} \alpha} \operatorname{VaRu}(\alpha)\right|_{\alpha=\varepsilon}
$$

Definition 2.6 (Tail value at ruin stability) Consider the perturbed compound Poisson risk process in Eq. 1 and its probability of ruin $\psi(x)=\mathbb{P}(T<\infty)$, for any $x \geq 0$. Assume $\psi$ is strictly decreasing at level $\varepsilon \in(0,1)$. Then the associated tail value at ruin stability at level $\varepsilon \in(0,1)$ is given by

$$
\operatorname{DTVaRu}(\varepsilon)=\left.\frac{\mathrm{d}}{\mathrm{d} \alpha} \mathbb{E}[L \mid L>\operatorname{VaRu}(\alpha)]\right|_{\alpha=\varepsilon} .
$$

We can note that $D \operatorname{VaRu}(\varepsilon) \leq 0$ and $D \operatorname{TVaRu}(\varepsilon) \leq 0$ for all $\varepsilon>0$.

Result 2.7 (Saddlepoint approximation to the $\mathrm{VaRu}$ stability) Assume $\psi$ is strictly decreasing at level $\varepsilon \in(0,1)$. Because Eq. 10 gives the initial capital $x$ as a function of the saddlepoint $v, r_{x}$ in Eq. 11 and $s_{x}$ in Eq. 12 can be re-expressed as functions of $v$ and in this case they are denoted $r(v)$ and $s(v)$, respectively. Then

$$
D \operatorname{VaRu}_{\mathrm{S}}(\varepsilon)=-\frac{a s\left(u_{k^{\star}}(1-\varepsilon)\right)}{u_{k^{\star}}(1-\varepsilon) \phi\left(r\left(u_{k^{\star}}(1-\varepsilon)\right)\right)}
$$


is a saddlepoint approximation to $D \operatorname{VaRu}(\varepsilon)$, where $u_{k^{\star}}(1-\varepsilon)$ is the NewtonRaphson approximation to the saddlepoint given by Eq. 18 in Result 2.3 and where

$$
a=\frac{1}{\sqrt{2 \pi}} \int_{-\infty}^{\bar{v}} \exp \left(K_{L}(v)-v K_{L}^{\prime} v\right) \sqrt{K_{L}^{\prime \prime}(v)} \mathrm{d} v
$$

is a normalizing constant.

Proof Let $\varepsilon>0$. From the differentiation formula of the inverse function, we obtain

$$
D \operatorname{VaRu}(\varepsilon)=\frac{1}{\psi^{\prime} \circ \operatorname{VaRu}(\varepsilon)} .
$$

Let $x \geq 0$. Because $-\psi^{\prime}(x)$ is a probability density at $x$, precisely the density of the maximal aggregate loss $L$, it can be approximated by the normalized saddlepoint approximation of Daniels (1954) as

$$
\frac{\exp \left(K_{L}\left(v_{x}\right)-x v_{x}\right)}{a \sqrt{2 \pi K_{L}^{\prime \prime}\left(v_{x}\right)}} .
$$

This latter expression can be re-formulated in terms of Eqs. 11 and 12 above as $v_{x} \phi\left(r_{x}\right) /\left(a s_{x}\right)$, which is then evaluated at $\operatorname{VaRu}_{\mathrm{S}}(\varepsilon)$ given by Eq. 19.

Result 2.8 (Saddlepoint approximation to the TVaRu stability) Assume $\psi$ is strictly decreasing at level $\varepsilon \in(0,1)$. Then a saddlepoint approximation to $D \operatorname{TVaRu}(\varepsilon)$ is given by

$$
D \operatorname{TVaRu}_{\mathrm{S}}(\varepsilon)=\frac{1}{\varepsilon}\left(\operatorname{VaRu}_{\mathrm{S}}(\varepsilon)-\operatorname{TVaRu}_{\mathrm{S}}(\varepsilon)\right)
$$

where $\operatorname{VaRu}_{\mathrm{S}}(\varepsilon)$ and $\mathrm{TVaRu}_{\mathrm{S}}(\varepsilon)$ are given by Eq. 19 in Result 2.3 and by Eq. 23 in Result 2.4, respectively.

Proof Let $\varepsilon \in(0,1)$. By differentiating the integral representation in Eq. 24 of $\mathrm{TVaRu}(\varepsilon)$ and by some further simplifications, we obtain

$$
D \operatorname{TVaRu}(\varepsilon)=\frac{1}{\varepsilon}(\operatorname{VaRu}(\varepsilon)-\operatorname{TVaRu}(\varepsilon))=-\frac{1}{\varepsilon^{2}} \int_{\operatorname{VaRu}(\varepsilon)}^{\infty} \psi_{\mathrm{S}}(x) \mathrm{d} x .
$$

The result simply follows after replacing $\operatorname{VaRu}(\varepsilon)$ and $\mathrm{TVaRu}(\varepsilon)$ by their saddlepoint approximations $\operatorname{VaRu}_{\mathrm{S}}(\varepsilon)$ and $\operatorname{TVaRu}_{\mathrm{S}}(\varepsilon)$.

\subsection{Numerical Comparisons}

In this section we illustrate the numerical accuracy of the four saddlepoint approximations presented in Section 2.1 by the following example. For the individual claim amounts we consider the linear combination of exponential distributions with CDF $F(x)=1-10 \mathrm{e}^{-x} / 9+\mathrm{e}^{-10 x} / 9$ and density $10\left(\mathrm{e}^{-x}-\mathrm{e}^{-10 x}\right) / 9$, for all $x \geq 0$. This is the CDF and the density of a sum of two independent exponentially distributed random variables with parameters 1 and 10. For this case, Dufresne and Gerber (1991, 


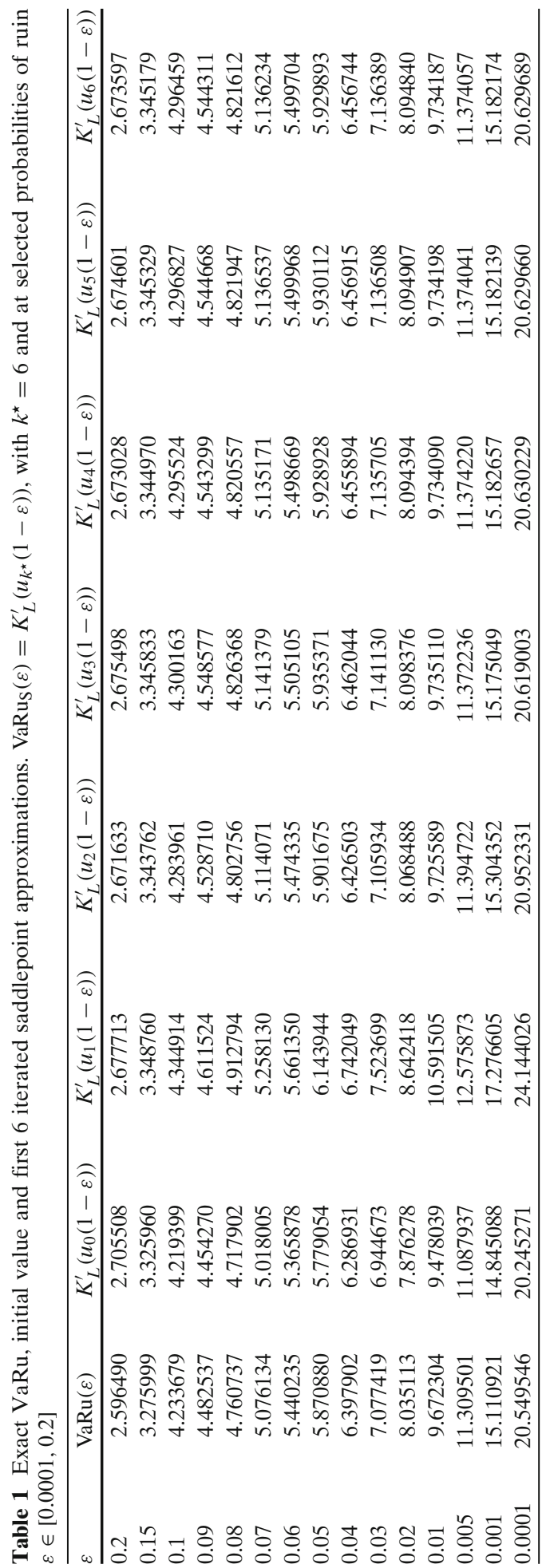


Table 2 Absolute and absolute relative errors of $\operatorname{VaRu}_{S}(\varepsilon)$ with $k^{\star}=6$ at selected probabilities of ruin $\varepsilon \in[0.0001,0.2]$, in the initial capital unit and in the probability scale

\begin{tabular}{llllll}
\hline$\varepsilon$ & $\left|\operatorname{VaRu}_{\mathrm{S}}(\varepsilon)-\operatorname{VaRu}(\varepsilon)\right|$ & $\frac{\left|\mathrm{VaRu}_{\mathrm{S}}(\varepsilon)-\operatorname{VaRu}(\varepsilon)\right|}{|\operatorname{VaRu}(\varepsilon)|}$ & $\psi\left(\operatorname{VaRu}_{\mathrm{S}}(\varepsilon)\right)$ & $\left|\psi\left(\operatorname{VaRu}_{\mathrm{S}}(\varepsilon)\right)-\varepsilon\right|$ & $\frac{|\psi(\operatorname{VaRu}(\varepsilon))-\varepsilon|}{\varepsilon}$ \\
\hline 0.2 & 0.077107 & 0.029697 & 0.193576 & 0.006424 & 0.032118 \\
0.15 & 0.069181 & 0.021117 & 0.145670 & 0.004331 & 0.028870 \\
0.1 & 0.062780 & 0.014829 & 0.097377 & 0.002623 & 0.026229 \\
0.09 & 0.061774 & 0.013781 & 0.087677 & 0.002323 & 0.025815 \\
0.08 & 0.060876 & 0.012787 & 0.077964 & 0.002036 & 0.025444 \\
0.07 & 0.060100 & 0.011840 & 0.068241 & 0.001759 & 0.025125 \\
0.06 & 0.059469 & 0.010931 & 0.058508 & 0.001492 & 0.024865 \\
0.05 & 0.059013 & 0.010052 & 0.048766 & 0.001234 & 0.024681 \\
0.04 & 0.058842 & 0.009197 & 0.039016 & 0.000984 & 0.024596 \\
0.03 & 0.058970 & 0.008332 & 0.029260 & 0.000740 & 0.024658 \\
0.02 & 0.059728 & 0.007433 & 0.019501 & 0.000499 & 0.024971 \\
0.01 & 0.061883 & 0.006398 & 0.009741 & 0.000259 & 0.025861 \\
0.005 & 0.064556 & 0.005708 & 0.004865 & 0.000135 & 0.026967 \\
0.001 & 0.071253 & 0.004715 & 0.000970 & 0.000030 & 0.029716 \\
0.0001 & 0.080143 & 0.003900 & 0.000097 & 0.000003 & 0.033363 \\
\hline
\end{tabular}

Section 6) provide an exact formula for the probability of ruin, which allows for numerical comparisons between the saddlepoint approximations and exact values. Thus, the individual claim amount MGF is $M_{X}(v)=10 /[(v-1)(v-10)]$ for all $v<1$, and the expected value is $\mu=1.1$. We consider $\sigma^{2}=0.4$ for the variance of the Wiener process, $\lambda=1$ for rate of the claim arrivals and $c=2$ for the premium rate.

Table 1 gives the exact $\operatorname{VaRu}(\varepsilon)$, the Cramér-Lundberg approximation $K_{L}^{\prime}\left(u_{0}(1-\right.$ $\varepsilon)$ ), and the iterations $K_{L}^{\prime}\left(u_{k}(1-\varepsilon)\right)$ for $k \in\{1, \ldots, 6\}$. We choose $k^{\star}=6$ because the maximum of $\left|u_{k}(1-\varepsilon)-u_{k-1}(1-\varepsilon)\right|$ for values of $\varepsilon$ in [0.0001,0.2] is smaller than the threshold $10^{-4}$ for $k=6$ and not for smaller values of $k$. (This maximum is $7.85 \cdot 10^{-5}$.) Thus $\operatorname{VaRu}_{\mathrm{S}}(\varepsilon)=K_{L}\left(u_{6}(1-\varepsilon)\right)$ is the saddlepoint approximation to $\operatorname{VaRu}(\varepsilon)$, for values of $\varepsilon$ in $[0.0001,0.2]$. Table 2 gives the absolute and relative errors

Table 3 Saddlepoint approximations $\operatorname{TVaRu}_{\mathrm{S}}(\varepsilon)$ with $k^{\star}=6$, absolute and absolute relative errors at selected probabilities of ruin $\varepsilon \in[0.0001,0.2]$

\begin{tabular}{|c|c|c|c|c|}
\hline$\varepsilon$ & $\operatorname{TVaRu}(\varepsilon)$ & $\mathrm{TVaRu}_{\mathrm{S}}(\varepsilon)$ & $\left|\operatorname{TVaRu}_{\mathrm{S}}(\varepsilon)-\operatorname{TVaRu}(\varepsilon)\right|$ & $\frac{\left|\operatorname{TVaRu}_{\mathrm{S}}(\varepsilon)-\mathrm{TVaRu}(\varepsilon)\right|}{|\mathrm{TVaRu}(\varepsilon)|}$ \\
\hline 0.2 & 4.958454 & 4.993622 & 0.035168 & 0.007093 \\
\hline 0.15 & 5.637949 & 5.670264 & 0.032316 & 0.005732 \\
\hline 0.1 & 6.595642 & 6.625961 & 0.030319 & 0.004597 \\
\hline 0.09 & 6.844500 & 6.874563 & 0.030063 & 0.004392 \\
\hline 0.08 & 7.122699 & 7.152572 & 0.029873 & 0.004194 \\
\hline 0.07 & 7.438095 & 7.467856 & 0.029761 & 0.004001 \\
\hline 0.06 & 7.802193 & 7.831942 & 0.029748 & 0.003813 \\
\hline 0.05 & 8.232830 & 8.262696 & 0.029866 & 0.003628 \\
\hline 0.04 & 8.759887 & 8.790052 & 0.030165 & 0.003444 \\
\hline 0.03 & 9.439382 & 9.470125 & 0.030744 & 0.003257 \\
\hline 0.02 & 10.397075 & 10.428892 & 0.031817 & 0.003060 \\
\hline 0.01 & 12.034263 & 12.068323 & 0.034060 & 0.002830 \\
\hline 0.005 & 13.671451 & 13.707996 & 0.036545 & 0.002673 \\
\hline 0.001 & 17.472886 & 17.515325 & 0.042439 & 0.002429 \\
\hline 0.0001 & 22.911520 & 22.961734 & 0.050215 & 0.002192 \\
\hline
\end{tabular}




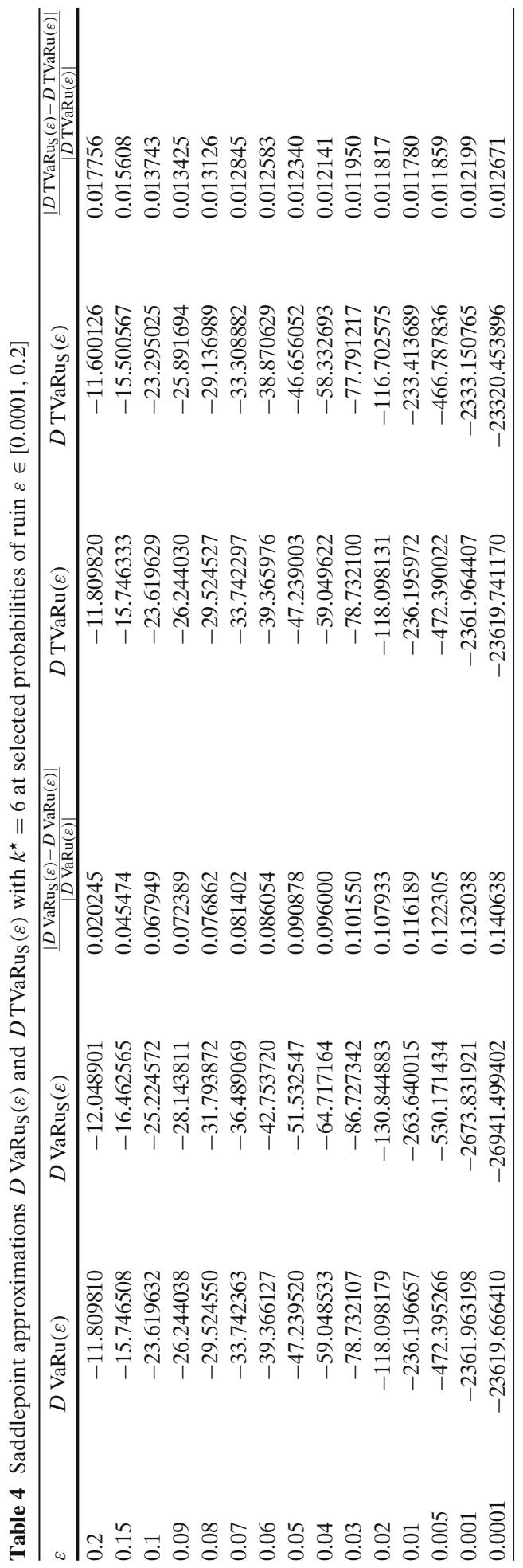


of the 6-step saddlepoint approximations $\operatorname{VaRu}_{S}(\varepsilon)$ shown in Table 1, in the unit of the initial capital, where the relative errors are standardized w.r.t. to the exact VaRu. We can observe that the absolute errors are small, between 0.05 and 0.08 , and that the absolute relative errors are very small and decrease as $\varepsilon$ decreases. In addition, $\psi\left(\operatorname{VaRu}_{\mathrm{S}}(\varepsilon)\right)$, the corresponding absolute errors $\left|\psi\left(\operatorname{VaRu}_{\mathrm{S}}(\varepsilon)\right)-\varepsilon\right|$ and the absolute relative errors $\left|\psi\left(\operatorname{VaRu}_{S}(\varepsilon)\right)-\varepsilon\right| / \varepsilon$ (i. e. the errors in the probability scale) are given for values of $\varepsilon$ in $[0.0001,0.2]$. This relative error appears bounded, typically below 0.03 , even for very small values of $\varepsilon$. So the iterative saddlepoint approximation $\mathrm{VaRu}_{\mathrm{S}}$ is very accurate. Table 3 gives the saddlepoint approximation $\mathrm{TVaRu}_{\mathrm{S}}(\varepsilon)$ based on $k^{\star}=6$ iterations to the exact $\operatorname{TVaRu}(\varepsilon)$, for values of $\varepsilon$ in $[0.0001,0.2]$. This table also provides absolute and relative errors in the unit of the initial capital, where the relative errors are standardized w. r. t. to the exact TVaRu. We can observe that the absolute errors are small, between 0.03 and 0.05 , and that the absolute relative errors are very small, between 0.002 and 0.007 . Table 4 gives the saddlepoint approximations $D \operatorname{VaRu}_{\mathrm{S}}(\varepsilon)$ and $D \mathrm{TVaRu}_{\mathrm{S}}(\varepsilon)$ based on $k^{\star}=6$ iterations to the corresponding exact stabilities for values of $\varepsilon$ in [0.0001, 0.2]. Relative errors are also shown. The saddlepoint approximation $D \mathrm{TVaRu}$ s shows very good accuracy, which is obviously related to the accuracies of $\mathrm{VaRu}_{\mathrm{S}}$ and $\mathrm{TVaRu}$. The absolute relative errors are between 0.01 and 0.02 . The saddlepoint approximation $D \mathrm{VaRu}_{\mathrm{S}}$ seems less precise, but still good. The absolute relative errors are between 0.02 and 0.14 . The value of the normalizing constant in Eq. 27 is $a=1.201883$.

\section{Probability of Ruin Due to Oscillation}

In Section 3.1 we develop two new numerical formulae for computing the probability of ruin due to claim and the probability of ruin due to perturbation. In Section 3.2 we illustrate the numerical accuracy of the proposed formulae by comparing them with formulae based on the saddlepoint approximation and on the FFT, which are derived by Gatto and Mosimann (2012).

\subsection{Recursive Formulae for the Probability of Ruin due to Oscillation}

As mentioned in the introduction, the probability of ruin $\psi$ can be decomposed into probability of ruin due to creeping $\psi^{(1)}$ and probability of ruin due to claim $\psi^{(2)}$, precisely $\psi(x)=\psi^{(1)}(x)+\psi^{(2)}(x)$ for all $x \geq 0$. Since the deficit at time of ruin is zero if it arises from creeping, ruin due to creeping is less problematic to the insurer than ruin due to claim, where the deficit at ruin is negative. The former case entails a temporary shortage of capital, which appears easier to overcome than the more substantial deficit induced by ruin due to claim. We propose a new extension of a recursive method for computing upper and lower bounds to the probability of ruin in the perturbed risk process in Eq. 1, proposed by Gatto and Mosimann (2012, Section 3), to the computation of the probability of ruin by creeping. Gatto and Mosimann (2012) generalize a method proposed by Dufresne and Gerber (1989) for the risk process without perturbation. This method discretizes the summands appearing in the decomposition in Eq. 4. By rounding up and down those summands, lower and upper bounds for $\psi$ can be constructed. These bounds converge towards 
the true value as the discretization degree becomes smaller. Denote by $\eta>0$ the discretization unit. The algorithm of Gatto and Mosimann (2012) is as follows.

Let

$$
h_{\mathrm{L} k}^{(j)}=\mathbb{P}\left(\left\lfloor L_{1}^{(j)} / \eta\right\rfloor=k\right)=H_{j}(\eta(k+1))-H_{j}(\eta k),
$$

for $k \in\{0,1, \ldots\}$, and

$$
h_{\mathrm{U} k}^{(j)}= \begin{cases}\mathbb{P}\left(\left[L_{1}^{(j)} / \eta\right]=k\right)=h_{\mathrm{L}(k-1)}^{(j)}, & \text { if } k \in\{1,2, \ldots\}, \\ 0, & \text { if } k=0,\end{cases}
$$

where $j \in\{1,2\}$. As usual, $\lfloor\cdot\rfloor$ is the floor function (i.e. the integer part) and $\lceil\cdot\rceil$ is the ceiling function (i. e. the smallest of all larger integers). Let us define the starting values

$$
f_{\mathrm{L} 0}=\frac{p h_{\mathrm{L} 0}^{(1)}}{1-(1-p) h_{\mathrm{L} 0}^{(1)} h_{\mathrm{L} 0}^{(2)}} \quad \text { and } \quad f_{\mathrm{U} 0}=0 .
$$

Then, the following values can be computed recursively,

$$
\begin{aligned}
& f_{\mathrm{L} i}=\frac{p h_{\mathrm{L} i}^{(1)}}{1-(1-p) h_{\mathrm{L} 0}^{(1)} h_{\mathrm{L} 0}^{(2)}}+\frac{1-p}{1-(1-p) h_{\mathrm{L} 0}^{(1)} h_{\mathrm{L} 0}^{(2)}} \sum_{k=1}^{i} f_{\mathrm{L}(i-k)}\left(\sum_{n=0}^{k} h_{\mathrm{L} n}^{(1)} h_{\mathrm{L}(k-n)}^{(2)}\right) \\
& f_{\mathrm{U} i}=p h_{\mathrm{U} i}^{(1)}+(1-p) \sum_{k=1}^{i} f_{\mathrm{U}(i-k)}\left(\sum_{n=0}^{k} h_{\mathrm{U} n}^{(1)} h_{\mathrm{U}(k-n)}^{(2)}\right)
\end{aligned}
$$

for $i \in\{1,2, \ldots\}$. This leads to the following lower and upper bounds for the probability of ruin,

$$
1-\sum_{i=0}^{\lceil x / \eta\rceil-1} f_{\mathrm{L} i} \leq \psi(x) \leq 1-\sum_{i=0}^{\lfloor x / \eta\rfloor} f_{\mathrm{U} i}
$$

for all $x>0$.

An approximation to $\psi(x)$ can be obtained by any intermediate value between the lower and upper bounds above, for example by

$$
\psi_{\mathrm{B}}(x)=1-\frac{1}{2}\left(\sum_{i=0}^{\lfloor x / \eta\rfloor} f_{\mathrm{L} i}+\sum_{i=0}^{\lceil x / \eta\rceil-1} f_{\mathrm{U} i}\right) .
$$

We can give the following remarks. The precision of the approximation in Eq. 36 can be improved by decreasing the discretization unit. The probabilities given by Eq. 31 are just the shifted probabilities given by Eq. 30 so that Eqs. 33, 34 could be re-expressed in terms of a single sequence of probabilities (after setting components with negative indices equal to 0 where applicable).

We now suggest using this method of upper and lower bounds for approximating the probabilities of ruin due to creeping and jump, which are $\psi^{(1)}$ and $\psi^{(2)}$. 
Result 3.1 Let $\eta>0$ be the discretization parameter used for $\psi_{\mathrm{B}}$ in Eq. 36. Then the formulae

$$
\psi_{\mathrm{B}}^{(1)}(x)=-\frac{1}{p \zeta} \frac{\psi_{\mathrm{B}}(x+\eta / 2)-\psi_{\mathrm{B}}(x-\eta / 2)}{\eta},
$$

for all $x \geq \eta / 2$, and

$\psi_{\mathrm{B}, \mathrm{R}}^{(1)}(x)=-\frac{1}{p \zeta}\left(\frac{9}{8} \frac{\psi_{\mathrm{B}}(x+\eta / 2)-\psi_{\mathrm{B}}(x-\eta / 2)}{\eta}-\frac{1}{8} \frac{\psi_{\mathrm{B}}(x+3 \eta / 2)-\psi_{\mathrm{B}}(x-3 \eta / 2)}{3 \eta}\right)$,

for all $x \geq 3 \eta / 2$, provide approximations to $\psi^{(1)}(x)=\mathbb{P}\left(T<\infty, Y_{T}=0\right)$ with vanishing errors as $\eta \rightarrow 0$.

Proof From Dufresne and Gerber (1991, Eqs. 4.3 and 4.5) follows that

$$
\psi^{(1)}(x)=-\frac{1}{p \zeta} \frac{\mathrm{d}}{\mathrm{d} x} \psi(x),
$$

for all $x \geq 0$. As $\psi^{(1)}$ is proportional to the derivative of $\psi$, we suggest applying Richardson's extrapolation to $\psi_{\mathrm{B}}$ given by Eq. 36. Let $g \in \mathscr{C}^{3}([a, b])$ and $x \in(a, b)$, for some $a<b \in \mathbb{R}$, then we can see that $g^{\prime}(x)=[g(x+h)-g(x-h)] /(2 h)+\mathrm{O}\left(h^{2}\right)$, as $h \downarrow 0$. Thus $g^{\prime}(x)$ can be approximated by the above Newton quotient with an error $\mathrm{O}\left(h^{2}\right)$, where $h>0$ is small. If $g \in \mathscr{C}^{5}([a, b])$, then this error can be reduced by using Richardson extrapolation, yielding

$$
g^{\prime}(x)=\frac{4}{3} \frac{g(x+h)-g(x-h)}{2 h}-\frac{1}{3} \frac{g(x+2 h)-g(x-2 h)}{4 h}+\mathrm{O}\left(h^{4}\right),
$$

as $h \downarrow 0$. With different sampling intervals, we obtain

$$
g^{\prime}(x)=\frac{9}{8} \frac{g(x+h / 2)-g(x-h / 2)}{h}-\frac{1}{8} \frac{g(x+3 h / 2)-g(x-3 h / 2)}{3 h}+\mathrm{O}\left(h^{4}\right),
$$

as $h \downarrow 0$. With both formulae above, the coefficients preceding the quotients sum up to one and are chosen such that the error terms of second order cancel. More detail on Richardson's extrapolation can be found e.g. in Kincaid and Cheney (2001). In our situation, we do not have access to the true probability of ruin but to approximation $\psi_{\mathrm{B}}$ given in Eq. 36. So we cannot expect that the total errors correspond to the ones stated above, as they depend on the accuracy of $\psi_{\mathrm{B}}$ in terms of $\eta$ as well. Nevertheless, the total errors do converge to zero as $\eta \rightarrow 0$. Also numerical studies indicate that Richardson's extrapolation in Eq. 38 does really improve the basic approximation in Eq. 37.

Note that since approximation in Eq. 36 is most accurate at lattice points $\{\eta k: k \in 0,1, \ldots\}$, it is advisable to apply the approximations $\psi_{\mathrm{B}}^{(1)}$ and $\psi_{\mathrm{B}, \mathrm{R}}^{(1)}$ as given in Result 3.1 to points that belong to the lattices $\{(2 k+1) \eta / 2: k \in\{0,1, \ldots\}\}$ and $\{(2 k+1) \eta / 2: k \in\{1,2, \ldots\}\}$, respectively. Although Richardson extrapolation is not feasible for $x<3 \eta / 2$ (due to the way the formula is constructed), it is still possible to set $\psi_{\mathrm{B}, \mathrm{R}}^{(1)}(x) \stackrel{\text { def }}{=} \psi_{\mathrm{B}}^{(1)}(x)$ for $x \in[\eta / 2,3 \eta / 2)$ and to set $\psi_{\mathrm{B}, \mathrm{R}}^{(1)}(0) \stackrel{\text { def }}{=} \psi_{\mathrm{B}}^{(1)}(0) \stackrel{\text { def }}{=} \psi^{(1)}(0)=$ 1. For $x \in(0, \eta / 2]$, neither of $\psi_{\mathrm{B}}^{(1)}$ and $\psi_{\mathrm{B}, \mathrm{R}}^{(1)}$ can be defined in a sensible way. 
3.2 Numerical Comparisons with Existing Methods

In this section we compare the approximations to the probability of ruin by creeping given in Section 3.1 with two alternative methods, the FFT and the saddlepoint approximation, which are provided by Gatto and Mosimann (2012). We start by summarizing these two alternative methods.

The FFT is due to Cooley and Tukey (1965) and provides a fast algorithm for computing the discrete Fourier transform. It is widely applied and available in many numerical software packages, including $R$ ( $R$ Development Core Team 2012). The FFT approximation to the probabilities of ruin can be found in Gatto and Mosimann (2012, Section 4) and can be summarized as follows. Let $\xi$ be large such that $F_{L}(\xi) \simeq 1$ (or, equivalently, $\psi(\xi) \simeq 0$ ) and $n \in\{2,3, \ldots\}$ such that the discretization unit $\eta=\xi / n$ is small. For $j \in\{1,2\}$ and $k \in\{0, \ldots, n-1\}$, let

$$
h_{k}^{(j)}=\mathbb{P}\left(\eta k<L_{1}^{(j)} \leq \eta(k+1)\right)=H_{j}\left(\frac{k+1}{n} \xi\right)-H_{j}\left(\frac{k}{n} \xi\right) .
$$

Compute the discrete Fourier transform of the vectors $\left(h_{0}^{(j)}, \ldots, h_{n-1}^{(j)}\right)$, for $j \in\{1,2\}$, using the FFT and denote them as $\left(\tilde{h}_{0}^{(j)}, \ldots, \tilde{h}_{n-1}^{(j)}\right)$, for $j \in\{1,2\}$. Determine the discrete Fourier transform of Eq. 5 by

$$
\tilde{w}_{k}=\sum_{i=0}^{\infty} p(1-p)^{i}\left(\tilde{h}_{k}^{(1)}\right)^{i+1}\left(\tilde{h}_{k}^{(2)}\right)^{i}=\frac{p \tilde{h}_{k}^{(1)}}{1-(1-p) \tilde{h}_{k}^{(1)} \tilde{h}_{k}^{(2)}},
$$

for $k \in\{0, \ldots, n-1\}$. Compute by FFT the inverse discrete Fourier transform of the vector $\left(\tilde{w}_{0}, \ldots, \tilde{w}_{n-1}\right)$ and denote it $\left(w_{0}, \ldots, w_{n-1}\right)$. Thus for $k \in\{0, \ldots, n-1\}$,

$$
\begin{gathered}
\psi_{\mathrm{FFT}}\left(\frac{k}{n} \xi\right)=\sum_{i=k}^{n-1} w_{i}, \\
\psi_{\mathrm{FFT}}^{(1)}\left(\frac{k}{n} \xi\right)=\frac{n}{\xi} \frac{\sigma^{2}}{2 \lambda \mu \beta} w_{k}
\end{gathered}
$$

and

$$
\psi_{\mathrm{FFT}}^{(2)}\left(\frac{k}{n} \xi\right)=\left(1-\frac{n}{\xi} \frac{\sigma^{2}}{2 \lambda \mu \beta}\right) w_{k}+\sum_{i=k+1}^{n-1} w_{i}
$$

are the FFT approximations to $\psi(k \xi / n), \psi^{(1)}(k \xi / n)$ and $\psi^{(2)}(k \xi / n)$, respectively.

Note that the resulting approximation is sensitive to the choice of $\xi$ : if it is chosen too small, then the aliasing error can be substantial, see Gatto and Mosimann (2012, Remark 5), whereas if it is chosen too large, then the computation time increases. Refer to Gatto and Mosimann (2012) for further details on this FFT.

The following saddlepoint approximations to the probability of ruin can be found in Gatto and Mosimann (2012, Section 2). The saddlepoint approximation to the probability of ruin due to oscillation based on the formula of Daniels (1954) is given by

$$
\psi_{\mathrm{S}}^{(1)}(x)=\frac{\sigma^{2} v_{x}}{2 a \lambda \mu \beta s_{x}} \phi\left(r_{x}\right)=\frac{v_{x}}{\operatorname{ap\zeta } s_{x}} \phi\left(r_{x}\right),
$$


for all $x \geq 0$, where $a$ is the normalizing constant given in Eq. 27. When combined with the saddlepoint approximation of Lugannani and Rice (1980) in Eq. 20, this yields the saddlepoint approximation of the probability of ruin caused by claims or jumps

$$
\psi_{\mathrm{S}}^{(2)}(x)=1-\Phi\left(r_{x}\right)-\left[\frac{1}{r_{x}}-\frac{1}{s_{x}}\left(1-\frac{v_{x}}{a p \zeta}\right)\right] \phi\left(r_{x}\right)
$$

for all $x \geq 0$, see Gatto and Mosimann (2012) for further details. Note that by replacing the saddlepoint approximation in Eq. 20 by the one in Eq. 21, we can obtain the following alternative formulae to Eqs. 43 and 42,

$$
\psi_{\mathrm{S}}^{(2) *}(x)=1-\Phi\left(r_{x}+\frac{1}{r_{x}} \log \frac{s_{x}}{r_{x}\left(1-\frac{v_{x}}{a p \zeta}\right)}\right)
$$

and

$$
\psi_{\mathrm{S}}^{(1) *}(x)=\Phi\left(r_{x}+\frac{1}{r_{x}} \log \frac{s_{x}}{r_{x}\left(1-\frac{v_{x}}{a p \zeta}\right)}\right)-\Phi\left(z_{x}\right),
$$

respectively. The approximations in Eqs. 44 and 45 are asymptotically equivalent to Eqs. 43 and 42 for large values of the initial reserve $x$ only. For small values of $x \geq 0$, Eqs. 44 and 45 can be very misleading. For small probabilities of ruin, $x$ is typically large and so Eqs. 44 and 45 are accurate.

We now provide a numerical comparison of the above methods using the same framework as in Section 2.2. The graph on the first row and first column of Fig. 1 gives the absolute errors $\left|\psi(x)-\psi_{\mathrm{FFT}}(x)\right|$ (solid line) and $\left|\psi^{(1)}(x)-\psi_{\mathrm{FFT}}^{(1)}(x)\right|$ (dashed line) of the FFT approximation, for initial capitals $x$ within the interval [0,10]. The graph on the first row and second column gives $\left|\psi(x)-\psi_{\mathrm{B}}(x)\right|$ (solid line) and $\mid \psi^{(1)}(x)-$ $\psi_{\mathrm{B}, \mathrm{R}}^{(1)}(x) \mid$ (dashed line), the errors for the methods of upper and lower bounds, for $x$ within the interval $[0,10]$. The graphs on the second row give the corresponding relative errors: $\left|\psi(x)-\psi_{\mathrm{FFT}}(x)\right| / \psi(x)$ (solid line) and $\left|\psi^{(1)}(x)-\psi_{\mathrm{FFT}}^{(1)}(x)\right| / \psi^{(1)}(x)$ (dashed line) are on the graph of the left column and $\left|\psi(x)-\psi_{\mathrm{B}}(x)\right| / \psi(x)$ (solid line) and $\left|\psi^{(1)}(x)-\psi_{\mathrm{B}, \mathrm{R}}^{(1)}(x)\right| / \psi^{(1)}(x)$ (dashed line) are on the graph of the right column. For the FFT we have $n=2^{11}$ and $\eta=0.01$ (i. e. $\xi=n \eta=20.48$, with $\psi(\xi)=$ $\left.1.7666 \cdot 10^{-8}\right)$, whereas for the method of upper and lower bounds we have $\eta=0.01$. We mainly see that for both methods the relative errors are small, even though they increase as the initial capital increases, i. e. as the probability of ruin vanishes. Approximations $\psi_{\mathrm{FFT}}^{(1)}(x)$ and $\psi_{\mathrm{B}, \mathrm{R}}^{(1)}(x)$ are inaccurate as $x$ becomes very close to zero. This is due to the fact that $\psi$ is steep in the vicinity of zero, and the discretization unit remains constant and thus too large in the vicinity of zero. So $\psi^{(1)}$, which from Eq. 39 amounts to the derivative of $\psi$, cannot be accurately approximated. The accuracy might be slightly improved by choosing a finer discretization, but the problem would 

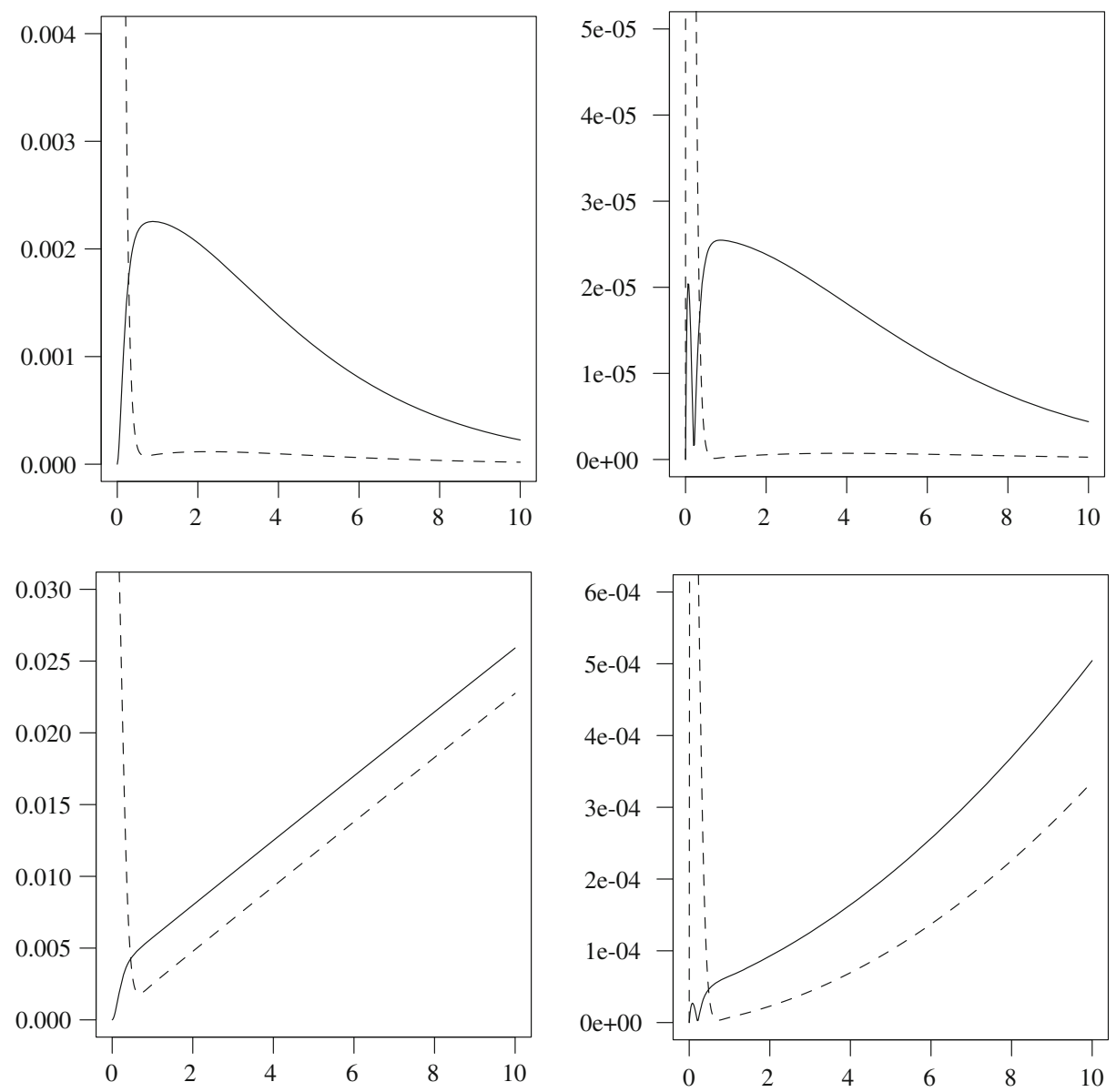

Fig. 1 Graph in the left column and top row: $\left|\psi-\psi_{\mathrm{FFT}}\right|$ with solid line and $\left|\psi^{(1)}-\psi_{\mathrm{FFT}}^{(1)}\right|$ with dashed line. Graph in the left column and bottom row: $\left|\psi-\psi_{\mathrm{FFT}}\right| / \psi$ with solid line and $\left|\psi^{(1)}-\psi_{\mathrm{FFT}}^{(1)}\right| / \psi^{(1)}$ with dashed line. Graph in the right column and top row: $\left|\psi-\psi_{\mathrm{B}, \mathrm{R}}\right|$ with solid line and $\left|\psi^{(1)}-\psi_{\mathrm{B}, \mathrm{R}}^{(1)}\right|$ with dashed line. Graph in the right column and bottom row: $\left|\psi-\psi_{\mathrm{B}, \mathrm{R}}\right| / \psi$ with solid line and $\mid \psi^{(1)}-$ $\psi_{\mathrm{B}, \mathrm{R}}^{(1)} \mid / \psi^{(1)}$ with dashed line

reappear as one gets closer to zero. From a practical point of view, large probabilities of ruin with initial capitals close to zero are not interesting. Note finally that in this situation it is known that $\psi(0)=\psi^{(1)}(0)=1$. Figure 2 shows the saddlepoint approximation to the probability of ruin $\psi_{\mathrm{S}}(x)$ (solid line) and to the probability of ruin due to claim $\psi_{\mathrm{S}}^{(1)}(x)$ (dashed line), for values of $x$ within the interval $[0,10]$. The right graph shows the alternative formulae $\psi_{\mathrm{S}}^{*}(x)$ (solid line) and $\psi_{\mathrm{S}}^{(1) *}(x)$ (dashed line), for values of $x$ within the interval $[0,10]$. A comparison between the left and the right graphs of Fig. 2 shows that $\psi_{\mathrm{S}}$ and $\psi_{\mathrm{S}}^{*}$ are practically identical, $\psi_{\mathrm{S}}^{(1) *}(x)$ is almost identical to $\psi_{\mathrm{S}}^{(1)}(x)$ for values of $x$ larger than approximately 1 , but $\psi_{\mathrm{S}}^{(1) *}(x)$ fails totally for values of $x$ smaller than approximately 1 . Note also that the exact counterparts of $\psi_{\mathrm{S}}$ (solid line) and $\psi_{\mathrm{S}}^{(1)}$ - are drawn with dotted lines in Fig. 2 and 

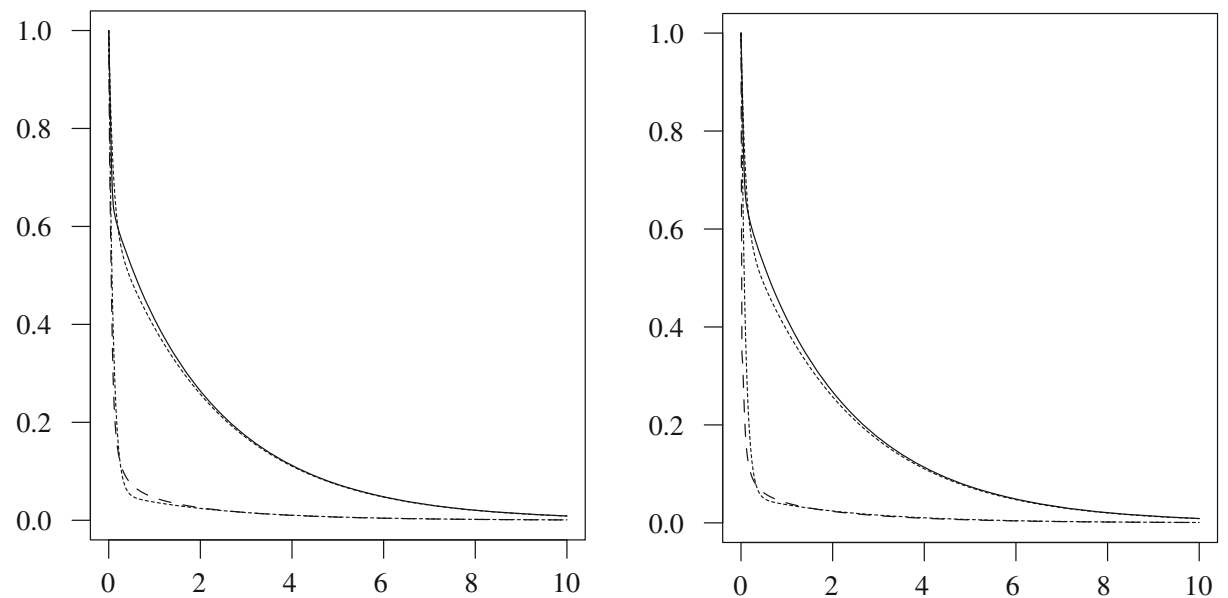

Fig. 2 Graph in the left column: $\psi_{\mathrm{S}}$ with solid line and $\psi_{\mathrm{S}}^{(1)}$ with dashed line. Graph in the right column: $\psi_{\mathrm{S}}^{*}(x)$ with solid line and $\psi_{\mathrm{S}}^{(1) *}(x)$ with dashed line. The corresponding exact values are shown with dotted lines

can be used for detailed interpretations of Figs. 1 and 3. The left graph of Fig. 3 shows the absolute errors $\left|\psi(x)-\psi_{\mathrm{S}}(x)\right|$ (solid line) and $\left|\psi^{(1)}(x)-\psi_{\mathrm{S}}^{(1)}(x)\right|$ (dashed line) of the saddlepoint approximations, for initial capitals $x$ in the interval $[0,10]$. The right graph shows the corresponding relative errors $\left|\psi(x)-\psi_{\mathrm{S}}(x)\right| / \psi(x)$ (solid line) and $\left|\psi^{(1)}(x)-\psi_{\mathrm{S}}^{(1)}(x)\right| / \psi^{(1)}(x)$ (dashed line). We see that the saddlepoint approximations have bounded relative errors, whereas this is not the case for the FFT and for the method of lower and upper bounds.
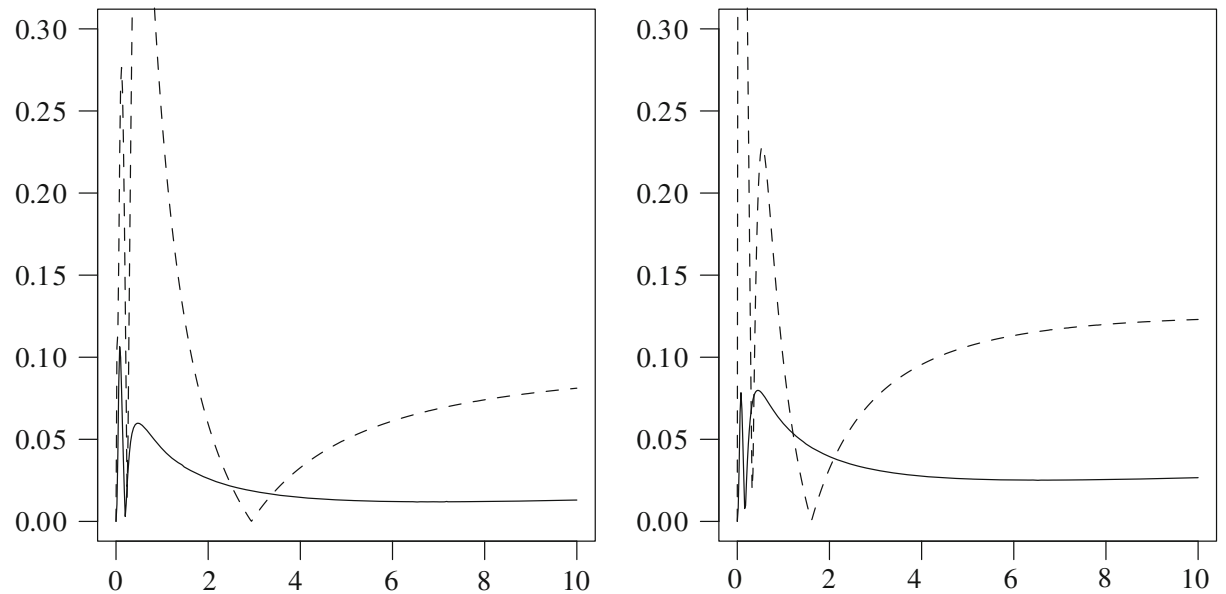

Fig. 3 Graph in the left column: $\left|\psi-\psi_{\mathrm{S}}\right| / \psi$ with solid line and $\left|\psi^{(1)}-\psi_{\mathrm{S}}^{(1)}\right| / \psi^{(1)}$ with dashed line. Graph in the right column: $\left|\psi-\psi_{\mathrm{S}}^{(*)}\right| / \psi$ with solid line and $\left|\psi^{(1)}-\psi_{\mathrm{S}}^{(1) *}\right| / \psi^{(1)}$ with dashed line 


\section{Heavy-Tailed Individual Claim Amounts and Conclusions}

In this concluding section we explain how the method of upper and lower bounds for computing the probabilities of ruin and the probability of ruin due to creeping, presented in Section 3.1, can be used as an alternative to the saddlepoint approximations for computing VaRu, TVaRu, $D$ VaRu and $D$ TVaRu presented in Section 2.1. Because the method of upper and lower bounds is not limited to light-tailed individual claim amounts, this method allows to compute these four values with risk processes having heavy-tailed individual claim amounts. $\mathrm{VaRu}$ and $\mathrm{TVaRu}$ can be obtained from $\psi_{\mathrm{B}}$ or $\psi_{\mathrm{B}, \mathrm{R}}$. We see from Eq. 28 that in order to compute $D \mathrm{VaRu}(\varepsilon)$ we need $-\psi^{\prime}(x)$. We see from Eq. 39 that $-\psi^{\prime}(x)=p \zeta \psi^{(1)}(x)$. Now $\psi^{(1)}(x)$, the probability of ruin by creeping, can be approximated by $\psi_{\mathrm{B}}^{(1)}$ or $\psi_{\mathrm{B}, \mathrm{R}}^{(1)}(x)$, as given by Eqs. 37 and 38 . So we can always replace the saddlepoint approximation, whose validity is restricted to light-tailed individual claim amount distributions, by the method of upper and lower bounds, which holds for heavy-tailed individual claim amounts.

The method of upper and lower bounds is most efficient when $\int_{0}^{x} 1-F(y) \mathrm{d} y$, for $x>0$, in the definition of $H_{2}$, given just after Eq. 5, can be solved analytically, as it happens with the exponential and the Pareto distributions for example. Otherwise, Gatto and Mosimann (2012, Remark 4) suggest the following discretization. Since $1-F$ is non-increasing, we can consider the following upper bounds,

$$
h_{\mathrm{L} k}^{(2)}=H_{2}(k+1)-H_{2}(k)=\frac{1}{\mu} \int_{k}^{k+1} 1-F(x) \mathrm{d} x \leq \frac{1}{\mu}(1-F(k)),
$$

for $k \in\{0,1, \ldots\}$, and the following lower bounds,

$$
h_{\mathrm{U} k}^{(2)} \geq \frac{1}{\mu}(1-F(k)) \text {, }
$$

for $k \in\{1,2, \ldots\}$. Substituting $(1-F(k)) / \mu$ for $h_{\mathrm{L} k}^{(2)}$ in Eqs. 32 and 33 leads to a lower bound for the probability of ruin, again by Eq. 35. Substituting $(1-F(k)) / \mu$ for $h_{\mathrm{U} k}^{(2)}$ in Eq. 34 leads to an upper bound for the probability of ruin, again by Eq. 35 .

The computer programs used for this article are written in $\mathrm{R}$ and can be obtained at http://cran.r-project.org/package=sdprisk.

Acknowledgements The authors thank the editor, an associate editor and two referees for several suggestions and corrections that improved the quality of this article. The authors are also grateful to the Swiss National Science Foundation for financial support (grant 200021-121901).

\section{References}

Artzner P, Delbaen F, Eber JM, Heath D (1997) Thinking coherently. Risk 10(11):68-71

Asmussen S, Rolski T (1991) Computational methods in risk theory: a matrix-algorithmic approach. Insur, Math Econ 10(4):259-274

Barndorff-Nielsen OE, Cox DR (1989) Asymptotic techniques for use in statistics. Monographs on statistics and applied probability, no 31. Chapman \& Hall/CRC

Barndorff-Nielsen OE, Schmidli H (1995) Saddlepoint approximation for the probability of ruin in finite time. Scand Actuar J (2):169-186

Cheridito P, Delbaen F, Kupper M (2006) Coherent and convex monetary risk measures for unbounded càdlàg processes. Finance Stoch 10(3):427-448

Cooley JW, Tukey JW (1965) An algorithm for the machine calculation of complex fourier series. Math Comput 19(90):297-301 
Daniels HE (1954) Saddlepoint approximations in statistics. Ann Math Stat 25(4):631-650

Dufresne F, Gerber HU (1989) Three methods to calculate the probability of ruin. ASTIN Bull 19(1):71-90

Dufresne F, Gerber HU (1991) Risk theory for the compound poisson process that is perturbed by diffusion. Insur Math Econ 10(1):51-59

Field C, Ronchetti E (1990) Small sample asymptotics, IMS lecture notes-monograph series, vol 13. Institute of Mathematical Statistics, Hayward, CA

Gatto R (2010) A saddlepoint approximation to the distribution of inhomogeneous discounted compound poisson processes. Methodol Comput Appl Probab 12(3):533-551

Gatto R (2011) Values and tail values at risk of doubly compound inhomogeneous and contagious aggregate loss processes. Math Comput Model 54(5-6):1523-1535.

Gatto R (2012) Saddlepoint approximations to tail probabilities and quantiles of inhomogeneous discounted compound poisson processes with periodic intensity functions. Methodol Comput Appl Probab 14(4):1053-1074

Gatto R, Mosimann M (2012) Four approaches to compute the probability of ruin in the compound poisson risk process with diffusion. Math Comput Model 55(3-4):1169-1185

Jensen JL (1992) The modified signed likelihood statistic and saddlepoint approximations. Biometrika 79(4):693-703

Jensen JL (1995) Saddlepoint approximations. Oxford statistical science series, no 16. Oxford University Press, USA

Kincaid D, Cheney W (2001) Numerical analysis: mathematics of scientific computing, 3rd edn. The Brooks/Cole Series in Advanced Mathematics, Thomson Learning

Klugman SA, Panjer HH, Willmot GE (2008) Loss models: from data to decisions, 3rd edn. Wiley Series in Probability and Statistics, Wiley, New York

Lugannani R, Rice S (1980) Saddle point approximation for the distribution of the sum of independent random variables. Adv Appl Probab 12(2):475-490

Neuts MF (1981) Matrix-geometric solutions in stochastic models: an algorithmic approach, reprinted and corrected edn. Phoenix Edition Series, Dover, Mineola, New York

R Development Core Team (2012) R: a language and environment for statistical computing. R Foundation for Statistical Computing, Vienna, Austria

Trufin J, Albrecher H, Denuit MM (2009) Properties of a risk measure derived from ruin theory. Geneva Risk Insur Rev 36(2):174-188

Wang S (1995) One-step saddlepoint approximations for quantiles. Comput Stat Data Anal 20(1):65-74 